We appreciate the response to this publication feature - and welcome all contributions. Contributions may be sent to Phil Oshel, our Technical Editor at:

$$
\begin{array}{ll}
\text { Mr. Phil Oshel } & \text { Tel.: (608)833-2885 } \\
\text { PO Box 620068 } & \text { Fax: (608)836-1969 }
\end{array}
$$

\section{Ceramic Powder Preparation for EM}

We have been preparing ceramic powder samples in our own analytical and testing laboratory for a number of years for our commercial clients. We have found that such samples:

1) Have to be vacuum embedded in order to make sure all the internal pores are infiltrated with the resin

2) Diamond knife ultramicrotomy is always required, because glass knives just won't "cut" it, and:

3) It is important to embed in a resin (typically an "Epon substitute" such as SPI-Pon ${ }^{\mathrm{TM}} 812$ ), that allows the possibility of varying the hardness of the final resin through a considerable range. This feature is sometimes important in terms of ending up with a block with the right properties (e.g. hardness).

4) If particles are formed in situ, and not as the result of grinding, then they should be sputter coated with (preferably) platinum prior to embedding, so that once in the TEM, one would always be able to keep track of the original outside surfaces. Gold will do if $\mathrm{Pt}$ is not available. This way one can determine the presence of a denser skin of different morphology.

Contrast can be a problem for resolving the pores of the smallest dimensions, so one would probably be wise to think about the use of "holey" support films on the TEM grids. (We now prefer to use the Quantifoil ${ }^{\mathrm{TM}}$ brand of holey carbon films because of their stability and absence of structure in the carbon film.)

Materials science diamond knives are best for this type of work, because these kinds of samples will immediately impart to the knife edge the kinds of fine striations one works so hard to remove in the production of a more expensive "life science" diamond knife. In other words, using a new life science knife on these kinds of samples (in our opinion) is quite wasteful economically.

Charles Garber, SPI Supplies cgarber@2spi.com

\section{Cleaning a Calibration Standard Grid}

We have found that casting a drop of Dow Corning Sylgard 184 (mixed according to directions) onto our grating, curing, then peeling off the cured silicone cleans the grating very thoroughly. We've done this to all of the 3 micron pitch gratings in the NT-MDT grating set TGSO2 without any damage to the gratings. As a bonus, this produces a nice replica of the grating.
I mix the Sylgard thoroughly according to the instructions, then let it sit for 15 to 30 minutes to let the bubbles dissipate. It is helpful to corral the Sylgard somewhat so it doesn't spill over (and under) the grating. I do this by chopping off the big end of a plastic pipette tip (about $4 \mathrm{~mm}$ long), so there is now a circular cone about $5 \mathrm{~mm}$ in diameter (which fits within the grating substrate edges). Now drip in small drops of the Sylgard until the cone is full. I then place it in an oven at $60^{\circ} \mathrm{C}$ to $75^{\circ} \mathrm{C}$ for at least 4 hours. I cure for at least the time specified in the cure schedule provided with the Sylgard for that temperature. I then simply hold the grating between two fingers and use the other hand to peel away the pipette tip plus Sylgard. The grating is now free of particles and many other things that can't be rinsed away. The Sylgard piece can be pushed out from the back to be used as a microcontact stamp.

By the way, it is still possible to do it without the corral, but removing the Sylgard can be more difficult. I've used plastic tweezers in this case with success, but with a greater chance of little bits of Sylgard remaining on the grating. These bits are usually easily removed by swishing in detergent solution and rinsing with deionized water.

This is the same stuff used by George Whitesides' group at Harvard (and many others) for microcontact printing. See for example Xia, Y.; Tien, J.; Qin, D.; Whitesides, G. M., Langmuir 1996, 12, 4033.

$$
\begin{gathered}
\text { Rick Workman, University of Arizona } \\
\text { rworkman@U.Arizona.EDU }
\end{gathered}
$$

\section{Serial Sections for TEM Without Using Glue}

The following comments are for people trying to cut serial sections with Spurr's. I routinely cut serial sections with Spurr's without resorting to any kind of glue. Probably the most important thing is how the block is faced. I cut the trapezoid with a really long $(0.6$ to $0.9 \mathrm{~mm})$ base, and the top of the trapezoid is almost the same length. What's really critical here is that those two cuts are parallel. The two sides of the trapezoid are very short $(0.1 \mathrm{~mm})$. I cut the sections with a diamond knife, using a Reichert Ultracut $E$ with a cutting speed of $0.8 \mathrm{~mm}$ per second and a thickness setting of $80 \mathrm{~nm}$. I am doing this in a small room with a blasting air vent in the ceiling, so I have cut up a cardboard box and stuck it into the ceiling tiles in such a way that the air is diverted away from the microtome. I can pick up 15 to 25 sections in a row on a $0.4 \times 2 \mathrm{~mm}$ copper slotted grid coated with just formvar or formvar/carbon. I have never been any good at making the support films myself, so I buy the slotted grids precoated. Before picking up the ribbon, I dip a stick into chloroform and wave that over the sections, and they expand (or relax, depending on your point of view). I have a bunch of self locking tweezers and after picking up the sections, I leave the grid with the sections on it locked in the tweezers until they dry (if a wet slotted grid is put down on filter paper, the film might break).

Timothy G. Schneider, Thomas Jefferson University Timothy.Schneider@mail.tju.edu

\section{Embedding Vibratome ${ }^{\mathrm{TM}}$ Sections for TEM}

When we want to embed Vibratome sections in a thin layer

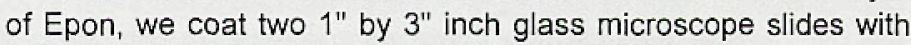
Liquid Release Agent (LRM, EMS Cat.\#70880), then place the 


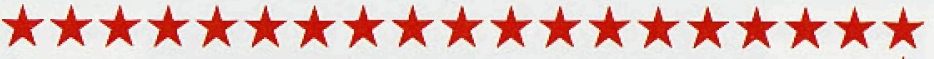
section and Epon in between the slides and cure. Our procedure for doing this is:

1) Acid clean and rinse the slides before use.

2) Dry in a drying oven overnight (the type used for drying cleaned laboratory glassware), then let cool to room temperature.

3) Dip slides in the LRA.

4) Stand slides upright and allow to dry in a dust-free area.

5) Tissue was sectioned at 50 to $100 \mu \mathrm{m}$ in a Vibratome, then processed into $100 \%$ epoxy. After the final change, rather than place in molds, place the sections on a coated slide, add some Epon, and then a second slide. The section is held flat by putting whatever weight is needed ( $5 \mathrm{~g}$ to $10 \mathrm{~g}$ typically; clips may also be used).

6) Place in the curing oven for the recommended time for the epoxy formulation used.

The Epon separates easily from the slides, and the residual LRA can be washed off because it is water soluble. (I understand this is a material that is used to release plastic parts from molds.) The clean slides are dipped in the liquid and allowed to dry ovenight before use. Slides can be washed in water and redipped. These embedded sections can be viewed in the light microscope after popping off one slide, and the appropriate part may be cut from the slab and remounted on a blank stub for thin sectioning.

We have used this technique for TEM identification of HRP labelled neurons.

John Chandler, Colorado State University chandler@lamar.colostate.edu

\section{A Brief Tip on Decolorizing}

DAB Staining for Immunohistochemistry

Diaminobenzidine (DAB) can be solubilized by incubating in weak ( $1 \%$ or less) bleach, which removes the orangebrown color. The bleach will completely remove the color if the slides reaction is allowed to run long enough. Run a test slide and check every 2 minutes at first in a microscope to get a handle on how long it will take for a given preparation. Acceptable endpoints could take less than 5 minutes or more than 2 hours depending on the particular section thickness, initial $\mathrm{DAB}$ intensity, etc. It may be best to just lighten the $\mathrm{DAB}$; and not decolorize it completely. Subsequent immunostaining success will depend on the tissue, antigen, and antibody, and the immunostaining may have to be modified to work with the $\mathrm{DAB}$ decolorization.

Subsequent immunostaining in my experience has not been affected, but in principle the bleaching process could oxidize an epitope so caveat lector.

Mike King, University of Florida College of Medicine making@nersp.nerdc.ufl.edu

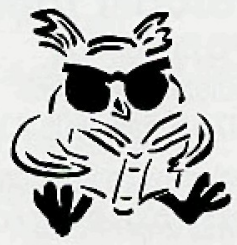

\section{Plan to attend ...}

\section{SCANNING 2001}

in New York City

Annual International Scientific Meeting sponsored by the

Foundation for Advances in Medicine and Science (FAMS) and SCANNING, The Journal of Scanning Microscopies

\section{May 5-7, 2001 at}

The Roosevelt Hotel 45th Street and Madison Avenue New York, NY, USA

\section{Welcome Reception - Saturday, May 5}

An international conference covering a wide range of topics related to the scanning microscopies with a forum for discussion and exchange of information. Upwards of two hundred papers will be presented in the areas of confocal microscopy, methodologies and new developments, applications of SEM in forensic science, food structure, probe microscopy including nanotechnology, pharmaceuticals, electron beam/instrument interaction modeling, materials, semi-conductor devices, and related areas. The program features Short Courses, invited and contributed scientific papers, posters, an exhibit hall showing the most advanced equipment and services available in SEM and related fields, and student award presentations.

\begin{tabular}{|c|c|}
\hline \multicolumn{2}{|c|}{ Program Committee: } \\
\hline $\begin{array}{l}\text { R.P. Becker } \\
\text { University of Illinois } \\
\text { Chicago, IL, USA }\end{array}$ & $\begin{array}{l}\text { D.C. Joy } \\
\text { University of Tennessee } \\
\text { Knoxville, TN, USA }\end{array}$ \\
\hline $\begin{array}{l}\text { P.C. Cheng } \\
\text { SUNY } \\
\text { Buffalo, NY, USA }\end{array}$ & $\begin{array}{l}\text { J.B. Pawley } \\
\text { University of Wisconsin } \\
\text { Madison, WI, USA }\end{array}$ \\
\hline $\begin{array}{l}\text { B.L. Giammara } \\
\text { Virtek Vision }\end{array}$ & $\begin{array}{l}\text { S.F. Platek } \\
\text { USFDA, Cincinnati, OH, USA }\end{array}$ \\
\hline Woburn, MA, USA & $\begin{array}{l}\text { M.T. Postek, Jr. } \\
\text { NIST, Gaithersburg, MD, USA }\end{array}$ \\
\hline $\begin{array}{l}\text { D. G. Howitt } \\
\text { University of California } \\
\text { Davis, CA, USA }\end{array}$ & $\begin{array}{l}\text { W.P. Wergin } \\
\text { USDA, Beltsville, MD, USA }\end{array}$ \\
\hline
\end{tabular}

\section{Call for papers ...}

Papers are now being solicited. Abstracts of no more than 700 words should be sent to SCANNING for publication in the Proceedings Issue. To obtain SCANNING 2001 Instructions for Abstracts, contact SCANNING/FAMS at the address below.

Abstract deadline - March 10, 2001.

For full program and registration information, contact:

Paula S. Pivnick at FAMS, Inc.

P.O. Box 832, Mahwah, NJ 07430-0832

Phone 201-818-1010 - Fax 201-818-0086

E-mail: scanning@fams.org

Internet: www.scanning.org 